\title{
The Neural Response in Short-Term Visual Recognition Memory for Perceptual Conjunctions
}

\author{
R. Elliott* and R. J . Dolan*, \\ *Wel I come Department of Cognitive Neurol ogy, Institute of Neurol ogy, 12 Queen Square, London WC1N 3BG, United Kingdom; \\ and †Royal FreeHospital School of Medicine, Rowland Hill Street, London, NW3 2PF, United Kingdom
}

Received J anuary 31, 1997

\begin{abstract}
Short-term visual memory has been widely studied in humans and animals using delayed matching paradigms. The present study used positron emission tomography (PET) to determine the neural substrates of delayed matching to sample for complex abstract patterns over a 5-s delay. More specifically, the study assessed any differential neural response associated with remembering individual perceptual properties (color only and shape only) compared to conjunction between these properties. Significant activations associated with short-term visual memory (all memory conditions compared to perceptuomotor control) were observed in extrastriate cortex, medial and lateral parietal cortex, anterior cingulate, inferior frontal gyrus, and thethalamus. Significant deactivations were observed throughout the temporal cortex. Although the requirement to remember color compared to shape was associated with subtly different patterns of blood flow, the requirement to remember perceptual conjunctions between these features was not associated with additional specific activations. These data suggest that visual memory over a delay of the order of $5 \mathrm{~s}$ is mainly dependent on posterior perceptual regions of the cortex, with the exact regions depending on the perceptual aspect of the stimuli to be remembered. 1998 Academic Press
\end{abstract}

\section{INTRODUCTION}

Most studies of visual memory involve complex, multidimensional stimuli. For example, in studies of object memory, the stimuli typically include col or, shape, and spatial location as well as inherent semantic properties. Even in studies of word recognition or recall, the stimuli include complex visual patterns as well as semantic and phonological dimensions. An effective application of functional imaging studies has been to unconfound different types of memory (e.g., semantic compared to episodic) and different subprocesses of memory (e.g., learning, storage, and retrieval). In this study, we used a simple visual recogni- tion task in an attempt to determine the neural mechanisms associated with short-term recognition of different perceptual dimensions of stimuli.

The study necessitated the design of complex stimuli, which could subsequently be distinguished on the basis of color alone, shape alone, or the conjunction between these two perceptual dimensions. This task is closely based on the delayed matching to sample (DMTS) paradigm which has been widely used in experimental animal studies and the stimuli were closely based on those used in the DMTS test from the CANTAB battery (e.g., Robbins et al., 1994). Importantly, it is a trialunique version of the paradigm such that all target stimuli are novel. In the typical DMTS experiment, animals are presented with an object and, after a variable delay period, must select the target object from a choice of alternatives. Successful performance of this task has been shown to depend on inferior temporal regions (Gross, 1973; Mishkin, 1982; Horel et al., 1987) as well as posterior perceptual cortex. There may also be an involvement of medial temporal lobe structures (Zola-Morgan and Squire, 1985; Mishkin and Murray, 1994 for review), particularly the rhinal cortex (ZolaMorgan et al., 1989; Murray, 1992; Meunier et al., 1993). However, it has been argued that medial temporal structures are only involved at relatively long delays (Alvarez et al., 1992, 1995). In human subjects medial temporal lobe damage typically results in longterm rather than short-term memory deficits (e.g., Milner, 1970). However, using the same paradigm as in the present study, Owen et al. (1995) found that neurosurgical patients with temporal lobe lesions or more restricted bilateral amygdalo-hippocampectomies showed pronounced impairments on this task, even at the shortest delays.

There have been numerous recent functional imaging studies of recognition memory processes, where there is no requirement to perform elaborative processing of stimuli or to process intervening material (e.g., J onides et al., 1993; Rol and and Gulyas, 1995; Goldberg et al., 1996; Baker et al., 1996; Owen et al., 1996). In all 
these studies, the memory conditions were associated with activation in a network of structures. The exact prefrontal and posterior cortical areas and subcortical structures activated by these studies depended on the nature of the stimulus input (abstract shapes, familiar, objects, spatial locations), the length of the delay, and the nature of the baseline condition.

While many of these studies implicitly considered memory for multidimensional stimuli, they did not explicitly address memory for conjunctions of visual features compared to single features alone. Functional imaging studies of perceptual conjunctions have been confined to the domain of attention. For example Corbetta et al. (1991) found that the requirement to divide attention between perceptual features rather than selectively attend particular features was associated with increased activation in the anterior cingulate and right dorsolateral prefrontal cortex. Rees et al. (1997) also found activation of the right dorsolateral prefrontal cortex when attention to conjunctions was compared to attention to features.

The present study aimed to extend the study of perceptual conjunctions to the mnemonic domain to assess the hypothesis that short-term visual memory is associated with activation within a network of posterior cortical (visual areas and inferior temporal cortex) and possibly prefrontal structures. On the basis of findings from studies of attention, we thought that memory for feature conjunction rather than individual features would be associated with predominantly rightsided, prefrontal activation.

\section{METHOD}

\section{Subjects}

Six right-handed male volunteers aged between 23 and 40 were recruited. Subjects with any neurological or psychiatric history were excluded. All subjects were given a structural MRI scan prior to PET scanning and the structural scan was checked to exclude abnormalities. This scan and the subjects' reported histories were considered sufficient evidence of normality. Five of the subjects were in full-time higher education and one was employed as a systems analyst, indicating an above average level of current cognitive functioning. The study was approved by thelocal hospital ethics committee, and permission to administer radioactive substances was obtained from the Administration of Radioactive Substances Advisory Committee (ARSAC) UK. Informed written consent was obtained prior to the study.

\section{Cognitive Activation Paradigm}

This experiment had a subtractive design with four experimental conditions. In all conditions subjects were presented with a test stimulus; all stimuli were complex shapes made up of four differently colored subelements (Fig. 1). After $2 \mathrm{~s}$ the test stimulus disappeared and was replaced by a blank screen. Five seconds later four choice stimuli were presented, one of which was the original stimulus, the spatial location of the correct stimulus varying randomly. Subjects had to identify the original stimulus by pressing the response button on a strip of four which corresponded to the spatial position of the correct stimulus. They were given $5 \mathrm{~s}$ in which to make this response (all subjects made all responses within this time) before the next problem appeared. Thus each trial took a total of $12 \mathrm{~s}$ and this was a fixed interval. During each scan, a run of nine problems, all from the same condition, were presented, beginning 30 $s$ before scanning. Subjects were scanned 12 times with three repetitions of each condition. The order of conditions was counterbalanced within and between subjects. Every individual problem was unique.

\section{Condition 1: Color Only}

In this condition, the shapes of the four choicestimuli were all the same as that of the test stimulus but the colors of the subelements differed. Therefore subjects could perform thetask by remembering the col ors of the test stimulus only.

\section{Condition 2: ShapeOnly}

In this condition, the col ors of the four choice stimuli were all the same as that of the test stimulus but the shapes of the subel ements differed. Therefore subjects could perform the task by remembering the shapes of the test stimulus only.

\section{Condition 3: Conjunction Condition}

In this condition, one of the incorrect choice stimuli had the same shapes as the test stimulus but different colors, one had the same col ors but different shapes and one had different col ors and shapes. Therefore, in order to perform this condition, subjects had to remember the

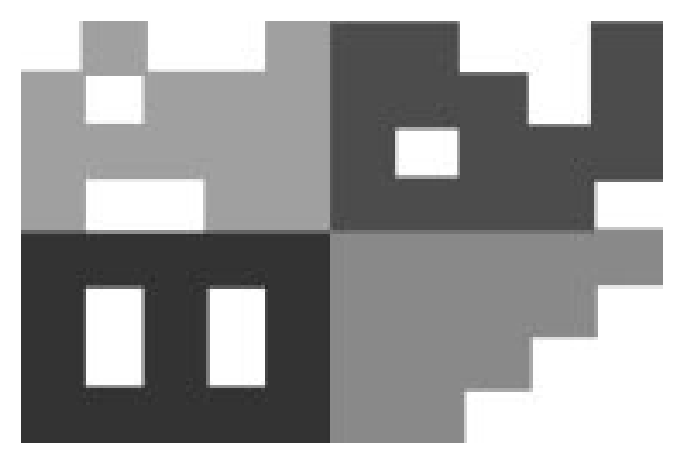

FIG. 1. An example of the test stimuli used. The four shades of grey represent four different colors. 
conjunction of color and shape in the test stimulus, since neither dimension alone uniquely defined the correct response.

\section{Condition 4: Perceptuomotor Control}

In this condition, all four "choice" stimuli were identical and had no elements in common with the test stimulus. Subjects were explicitly instructed not to attempt to remember the test stimulus and were told to respond to the appearance of the four "choice stimuli" by pressing a prespecified response button which was the same for the duration of the run. This control condition was chosen to match the perceptual and motor demands of the memory task as closely as possible. A consequence of this was to introduce certain possible confounds which cannot be explicitly addressed in this study. First, it is not possible to determine empirically the extent to which subjects complied with theinstruction not to remember the initial stimuli. Second, this condition requires subjects to remember which of four buttons to press, the effect of which cannot be directly studied. However, these are minor concerns which we did not consider to be critical.

\section{PET Scanning Technique}

Regional cerebral blood flow was measured with an ECAT HR + scanning system (CTI Siemens, Knoxville, TN) in 3D mode with septa retracted. For each scan, $555 \mathrm{Mbq}$ of $\mathrm{H}_{2}{ }^{15} \mathrm{O}$ were flushed through a venous cannula in the left antecubital vein with normal saline over $20 \mathrm{~s}$ at a rate of $10 \mathrm{ml} / \mathrm{min}$ by an automatic pump. After a delay of about $35 \mathrm{~s}$, a rise in counts could be detected at the head, peaking 30-40 s later, varying for individual subjects. The data were acquired during one 90-s frame, beginning $5 \mathrm{~s}$ before the rising phase of the head curve. A total of 12 scans were performed at intervals of $8 \mathrm{~min}$. Correction for attenuation was made by performing a transmission scan with an exposed 68 Ge/68 Ga external ring source before each session. I mages were reconstructed by filtered back projection to give a resolution of approximately $6 \mathrm{~mm}$ at full width half maximum and displayed in a $128 \times 128$ pixel format with 43 planes rendering the voxels approximately cubic.

\section{Data Analysis}

Data were analyzed using Statistical Parametric Mapping (SPM96, Well come Dept. of Cognitive Neurology, London, UK). Scans were realigned using the first as a reference and were subsequently transformed into a standard space corresponding to thestereotactic atlas of Talairach and Tournoux (1988). These normalized images were smoothed with a 12-mm FWHM isotropic Gaussian kernel.

This experiment had a subtractive design, in which conditions could be compared to each other. Analysis was carried out using the general linear model. The conditions for each subject were specified in the appropriate design matrix which also included gl obal activity as a confounding covariate and can therefore be considered an ANCOVA. Effects at each and every voxel were estimated according to the general linear model and regionally specific effects were compared using linear contrasts. The resulting set of voxel values for each contrast constituted a statistical parametric map of the t statistic (SPM $\{t\}$ ) which was then transformed to the unit normal distribution, SPM $\{Z\}$. Statistical inferences were based on the theory of random Gaussian fields (Friston et al., 1995).

\section{RESULTS}

\section{Performance Data}

The mean percentage of problems solved correctly and the mean response latencies under the three memory conditions are shown in Table 1 . This table shows that the conjunction condition was not significantly more difficult than the feature only conditions. Interestingly, five of the six subjects reported finding the shape only condition the most difficult and in fact, performance on this condition was better than for color only or conjunction.

The use of a perceptuomotor control task rather than rest as the baseline condition in this task means that the changes in rCBF which can be reported must be thought of as relative activations or deactivations. For example a deactivation associated with memory compared to control says only that blood flow is lower in the memory than the control condition. Whether this is due to reduced blood flow in the memory task or increased blood flow in the control task in relation to a rest state cannot be addressed by the present design. Therefore therCBF changes described bel ow should all bethought of as relative differences in blood flow associated with the different conditions.

\section{rCBF Changes Associated with Memory Conditions Compared to Control}

This comparison combines the three different memory conditions and compares them to the control condition

\section{TABLE 1}

Performance of Subjects Under the Three Memory Conditions

\begin{tabular}{lccc}
\hline & Color only & Shape only & Conjunction \\
\hline Percentage correct & 83 & 91 & 85 \\
Latency (ms) & 4385 & 3588 & 4015 \\
\hline
\end{tabular}


in order to determinethe general overall regions associated with recognition of colored shapes (Fig. 2).

Significant $(P<0.001)$ rCBF increases (relative activations) were observed mainly in posterior cortical regions (Table 2). Specific regions activated included the superior, medial, and inferior occipital gyri bilaterally (BA 18 and 19), the left inferior parietal lobe (BA 40), the posterior lateral portion of the precuneus bilaterally (BA 7), the fusiform gyrus (BA 37), and lingual gyrus bilaterally (BA 17/18). Frontal cortex areas activated were regions of the left premotor cortex (BA 4 and 6) extending to the anterior cingulate (BA 32) and the left inferior (BA 44) frontal gyrus. Subcortical

\section{TABLE 2}

Coordinates of Maximal Significant Changes of $\mathrm{rCBF}$ Between Memory Conditions and Perceptuomotor Control

\begin{tabular}{|c|c|c|c|c|c|c|}
\hline \multirow[b]{2}{*}{ Region of activation } & \multirow{2}{*}{$\begin{array}{l}\text { Left/ } \\
\text { right }\end{array}$} & \multirow{2}{*}{$\begin{array}{l}\text { Brodmann's } \\
\text { area }\end{array}$} & \multicolumn{3}{|c|}{$\begin{array}{l}\text { Talairach } \\
\text { coordinates }\end{array}$} & \multirow{2}{*}{$\begin{array}{c}\text { Z } \\
\text { value }\end{array}$} \\
\hline & & & $x$ & $y$ & z & \\
\hline \multicolumn{7}{|l|}{ rCBF increases } \\
\hline \multirow[t]{2}{*}{ Medial occipital gyrus } & $\mathrm{R}$ & 19 & 32 & -84 & 8 & 5.96 \\
\hline & L & 19 & -34 & -88 & 2 & 5.93 \\
\hline \multirow[t]{2}{*}{ Inferior occipital gyrus } & $\mathrm{L}$ & 18 & -32 & -84 & -10 & 5.61 \\
\hline & $\mathrm{R}$ & 18 & 38 & -76 & -6 & 4.34 \\
\hline \multirow[t]{2}{*}{ Superior occipital gyrus } & $\mathrm{L}$ & 19 & -30 & -72 & 34 & 5.51 \\
\hline & $\mathrm{R}$ & 19 & 32 & -78 & 28 & 5.23 \\
\hline \multirow[t]{2}{*}{ Lingual gyrus } & $\mathrm{R}$ & 17 & 20 & -90 & -14 & 5.19 \\
\hline & $\mathrm{L}$ & 17 & -16 & -90 & 4 & 4.17 \\
\hline Inferior parietal cortex & L & 40 & -32 & -50 & 56 & 4.68 \\
\hline \multirow[t]{2}{*}{ Precuneus } & $\mathrm{L}$ & 7 & -22 & -62 & 52 & 4.73 \\
\hline & $\mathrm{R}$ & 7 & 24 & -72 & 48 & 5.51 \\
\hline \multirow[t]{3}{*}{ Cerebellum } & $\mathrm{R}$ & & 0 & -68 & -24 & 5.21 \\
\hline & $\mathrm{L}$ & & -42 & -62 & -20 & 4.74 \\
\hline & L & & -22 & -46 & -26 & 4.24 \\
\hline \multirow[t]{2}{*}{ Fusiform gyrus } & $\mathrm{L}$ & 19/37 & -46 & -56 & -10 & 4.34 \\
\hline & $\mathrm{R}$ & 37 & 44 & -60 & -12 & 3.73 \\
\hline Inferior frontal gyrus & L & 44 & -42 & 14 & 26 & 4.64 \\
\hline Thalamus & $\bar{L}$ & & -16 & -14 & 4 & 4.41 \\
\hline Premotor cortex & L & 6 & -20 & 2 & 48 & 4.32 \\
\hline Anterior cingulate & $\mathrm{L}$ & 32 & -2 & 8 & 52 & 4.16 \\
\hline \multicolumn{7}{|l|}{ rCBF decreases } \\
\hline \multirow[t]{3}{*}{ Superior temporal gyrus } & $\mathrm{R}$ & 22 & 56 & -44 & 8 & 7.10 \\
\hline & $\mathrm{R}$ & 39 & 60 & -56 & 30 & 6.45 \\
\hline & L & 39 & -50 & -62 & 22 & 5.85 \\
\hline \multirow[t]{2}{*}{ Medial temporal gyrus } & $\mathrm{L}$ & 21 & -62 & -16 & -14 & 6.26 \\
\hline & $\mathrm{R}$ & 22 & 46 & -14 & 4 & 8.42 \\
\hline \multirow[t]{2}{*}{ Inferior temporal cortex } & L & 20 & -62 & -16 & -20 & 5.31 \\
\hline & $\mathrm{R}$ & 20 & 56 & 6 & -28 & 4.88 \\
\hline Hippocampus & $\mathrm{R}$ & & 20 & -12 & -18 & 4.44 \\
\hline Amygdala & $\mathrm{L}$ & & -22 & -6 & -16 & 4.64 \\
\hline Anterior frontal cortex & L & 9 & -2 & 54 & 22 & 6.08 \\
\hline Superior frontal gyrus & $\mathrm{R}$ & 9 & 20 & 52 & 30 & 5.80 \\
\hline \multirow[t]{2}{*}{ Cerebellum } & $\mathrm{L}$ & & -26 & -76 & -34 & 5.46 \\
\hline & $\mathrm{R}$ & & 26 & -90 & -36 & 5.17 \\
\hline \multirow[t]{2}{*}{ Precuneus } & $\mathrm{R}$ & 7 & 2 & -54 & 34 & 4.22 \\
\hline & L & 7 & -6 & -58 & 32 & 4.06 \\
\hline Putamen & $\mathrm{R}$ & & 28 & 2 & 8 & 3.88 \\
\hline
\end{tabular}

Note $Z=3.09$ for $P<0.001$. areas activated were the left thalamus and medial and lateral regions of the cerebellum bilaterally.

Significant $(P<0.001)$ rCBF decreases (relative deactivations) were observed throughout the temporal cortices bilaterally; in the superior (BA 22 and 39), medial (BA 21 and 39), and inferior (BA 20) temporal gyri (see Table 2). There were also deactivations, relative to control, in the anterior frontal cortex (BA 9) extending ventrally to the orbitofrontal cortex (BA 11 and 25 ), the right superior frontal gyrus (BA 8), and the left medial frontal gyrus (BA 8). Deactivations were also observed in the precuneus bilaterally with foci anterior and ventromedial to the foci of the activation. Subcortical deactivations were seen bilaterally in the posterior cerebellum, in the left amygdala, right hippocampus, and the right putamen.

\section{rCBF Changes Revealed Through Comparisons}

Between Different Memory Conditions (Table 3)

Conjunction compared to colour only. The only significant $(P<0.001)$ activation associated with memory based on conjunctions compared to memory for color alone was in the depths of the sulcus separating the inferior parietal and superior temporal cortices. Significant activations associated with memory for col or alone compared to conjunction were observed in the inferior frontal gyrus bilaterally (BA 44/45), the right superior temporal gyrus (BA 22), left medial temporal gyrus (BA 21), and left hippocampus.

Conjunction compared to shape only. The only significant $(P<0.001)$ activation associated with memory based on conjunction compared to memory for shape alone was in the ventrolateral cerebellum. Significant activations associated with memory for shape alone compared to conjunction were observed in the right inferior frontal gyrus (BA 44/45), left premotor cortex (BA 6), and anterior cingulate on the right (BA 32). Posterior activations associated with shape al one compared to conjunction were observed in the right superior temporal gyrus (BA 22), left inferior temporal cortex (BA 20), right medial occipital gyrus (BA 18), right inferior parietal cortex (BA 40), and anterior medial precuneus (BA 7) on the right.

Conjunction compared to singl efeatures only (Fig. 3). The sole activation associated with memory based on conjunction compared to memory for single features (color and shape conditions combined) was in the ventrolateral cerebellum. Significant activations associated with memory for single features compared to conjunction were observed in the right inferior frontal gyrus (BA 44/45), left premotor cortex (BA 6), and right anterior cingulate (BA 32). Posterior activations were observed in regions of the right superior temporal gyrus (BA 22), the left inferior temporal gyrus (BA 20), the anterior medial precuneus (BA 7) on the right and the left lingual gyrus (BA 18). There was also a 


\section{TABLE 3}

Coordinates of Maximal Significant Changes of rCBF Between Different Memory Conditions

\begin{tabular}{|c|c|c|c|c|c|c|}
\hline \multirow[b]{2}{*}{ Region of activation } & \multirow{2}{*}{$\begin{array}{l}\text { Left/ } \\
\text { right }\end{array}$} & \multirow{2}{*}{$\begin{array}{l}\text { Brodmann's } \\
\text { area }\end{array}$} & \multicolumn{3}{|c|}{$\begin{array}{l}\text { Talairach } \\
\text { coordinates }\end{array}$} & \multirow{2}{*}{$\begin{array}{c}\mathrm{Z} \\
\text { value }\end{array}$} \\
\hline & & & $x$ & $\mathrm{y}$ & $z$ & \\
\hline \multicolumn{7}{|l|}{ Conjunction-color } \\
\hline $\begin{array}{l}\text { Sulcus separating infe- } \\
\text { rior parietal and supe- } \\
\text { rior temporal cortices }\end{array}$ & $\mathrm{L}$ & & -30 & -34 & 26 & 3.90 \\
\hline \multicolumn{7}{|l|}{ Color-conjunction } \\
\hline \multirow[t]{2}{*}{ Inferior frontal gyrus } & $\mathrm{R}$ & $44 / 45$ & 46 & 16 & 16 & 4.66 \\
\hline & L & 44 & -54 & 10 & 10 & 3.48 \\
\hline Superior temporal gyrus & $\mathrm{R}$ & 22 & 44 & -48 & 20 & 3.93 \\
\hline Medial temporal gyrus & $\mathrm{L}$ & 21 & -48 & -44 & 2 & 3.57 \\
\hline Hippocampus & L & & -36 & -12 & -8 & 3.48 \\
\hline \multicolumn{7}{|l|}{ Conjunction-shape } \\
\hline Ventrolateral cerebellum & $\mathrm{L}$ & & -48 & -60 & -46 & 3.49 \\
\hline \multicolumn{7}{|l|}{ Shape-conjunction } \\
\hline Superior temporal gyrus & $\mathrm{R}$ & 22 & 44 & -48 & 20 & 5.44 \\
\hline Inferior temporal cortex & $\mathrm{L}$ & 20 & -40 & -10 & -18 & 3.83 \\
\hline Medial occipital cortex & $\mathrm{R}$ & 18 & 24 & -86 & 20 & 4.54 \\
\hline Inferior parietal cortex & $\mathrm{R}$ & 40 & 64 & -28 & 44 & 4.05 \\
\hline Precuneus & $\mathrm{R}$ & 7 & 10 & -58 & 30 & 3.89 \\
\hline Premotor cortex & L & 6 & -20 & 4 & 52 & 4.16 \\
\hline Anterior cingulate & $\mathrm{R}$ & 32 & 18 & 10 & 44 & 4.13 \\
\hline Inferior frontal cortex & $\mathrm{R}$ & $44 / 45$ & 46 & 16 & 16 & 3.80 \\
\hline \multicolumn{7}{|l|}{ Conjunction-features } \\
\hline Ventrolateral cerebellum & $\mathrm{L}$ & & -48 & -60 & -44 & 3.09 \\
\hline \multicolumn{7}{|l|}{ Features-conjunction } \\
\hline Superior temporal gyrus & $\mathrm{R}$ & 22 & 44 & -48 & 20 & 5.31 \\
\hline Hippocampus & L & & -36 & -12 & -10 & 3.72 \\
\hline Inferior temporal cortex & $\mathrm{L}$ & 20 & -30 & -20 & -32 & 3.58 \\
\hline Precuneus & $\vec{R}$ & 7 & 10 & -56 & 30 & 3.58 \\
\hline Lingual gyrus & L & 18 & -2 & -68 & 6 & 3.52 \\
\hline Inferior frontal gyrus & $\mathrm{R}$ & $44 / 45$ & 46 & 16 & 16 & 4.79 \\
\hline Premotor cortex & L & 6 & -20 & 6 & 52 & 3.67 \\
\hline Anterior cingulate & $\mathrm{R}$ & 32 & 20 & 10 & 44 & 3.38 \\
\hline \multicolumn{7}{|l|}{ Color-shape } \\
\hline Inferior frontal gyrus & L & 44 & -26 & 20 & 50 & 3.18 \\
\hline Lingual gyrus & $\mathrm{L}$ & 18 & -16 & -94 & -8 & 3.11 \\
\hline \multicolumn{7}{|l|}{ Shape-color } \\
\hline Anterior cingulate & $\mathrm{R}$ & 32 & 16 & 4 & 44 & 4.30 \\
\hline Inferior parietal cortex & $\mathrm{R}$ & 40 & 66 & -28 & 44 & 4.57 \\
\hline \multirow[t]{2}{*}{ Medial occipital cortex } & $\mathrm{R}$ & 19 & 22 & -86 & 24 & 4.21 \\
\hline & L & 19 & -20 & -74 & 34 & 4.08 \\
\hline Precuneus & $\mathrm{R}$ & 7 & 2 & -78 & 54 & 3.66 \\
\hline Cerebellum & $\mathrm{R}$ & & 14 & -50 & -6 & 3.96 \\
\hline
\end{tabular}

Note $Z=3.09$ corresponds to $P<0.001$.

significant activation in the left hippocampus which, adjusted blood flow values showed, was due to relatively decreased blood flow in the conjunction condition compared to the other three conditions (Fig. 3b).

Color compared to shape. The only significant $(\mathrm{P}<0.001)$ activations associated with memory for color alone compared to memory for shape alone were in the left inferior frontal gyrus (BA 44) and the left lingual gyrus (BA 18). Significant activations associated with memory for shape only compared to col or only were seen in the right anterior cingulate (BA 32), right inferior parietal cortex (BA 40), regions of the medial occipital gyrus (BA 19) bilaterally, posterior medial precuneus (BA 7), and right cerebellum.

\section{DISCUSSION}

This study demonstrated that an extensive network of mainly posterior cortical structures are involved in short-term visual memory for abstract patterns. The requirement to remember different perceptual features of the stimuli was associated with subtle differences in activations within this network. Interestingly memory for feature conjunction was not associated with specific activations relativeto single feature conditions, whereas memory for a particular feature was associated with specific activations relative to the conjunction condition.

The involvement of visual areas in short-term memory for abstract patterns is not a novel observation. In a study of memory for complex geometric patterns, recognition of previously learned patterns compared to rest involved activation of 18 overlapping fields of the occipital and parietal cortex (Roland and Gulyas, 1995). Activation of many of the same areas in the present study suggests that these regions are involved in memory rather than simply perception of stimuli, since the control task was perceptually matched. However, it should be noted that the memory conditions were likely to be associated with more eye-movements than the control condition. These were not measured explicitly and are a potential confound of the study design. The posterior cortical activations in the present study are far more extensive than those reported in studies of delayed response (J onides et al., 1993; Baker et al., 1996) which probably reflects the much greater complexity of the stimuli in the present study.

Prefrontal activations associated with the requirement to remember visual stimuli in this study are confined to the premotor cortex, anterior cingulate, and inferior frontal gyrus (BA 44) all on the left. The left-sided premotor activation cannot be due simply to the execution of a right-hand motor response since the same response is made in the control task. In the control task, however, the response is fully prespecified, whereas in the memory conditions the spatial location of the response is only determined when the choice of stimuli appear. This premotor activation may represent preparatory activity associated with a response which is not fully specified. It is consistent with activations observed in other studies with delayed response paradigms (J onides et al., 1993; Goldberg et al., 1996; Baker et al., 1996). Activity in the anterior cingulate has been associated with many paradigms requiring sel ective attention and response selection (Pardo et al., 1990; Friston et al., 1991; Corbetta et al ., 1993), includ- 
a

sagittal

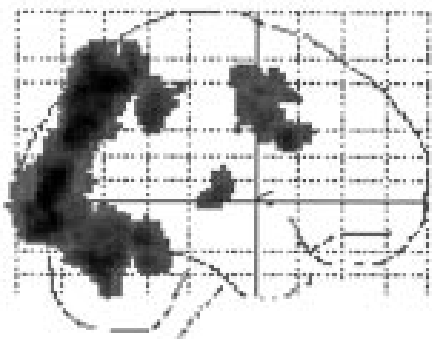

transverse

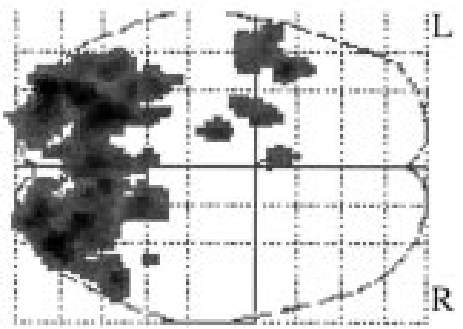

b coronal

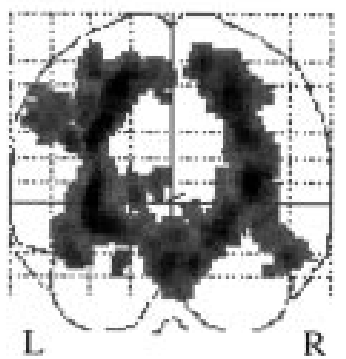

$\mathrm{R}$ sagittal

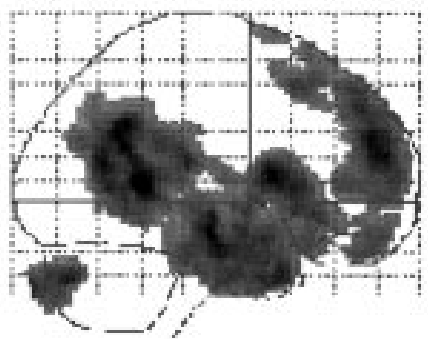

transverse coronal

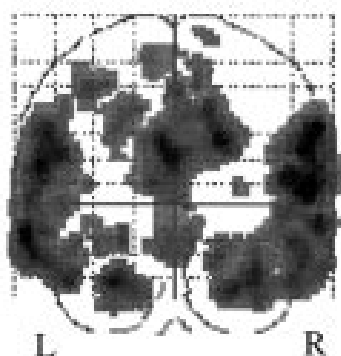

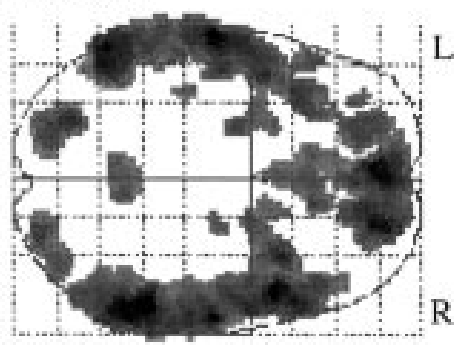

FIG. 2. (a) The activations associated with all three memory conditions compared to the control condition in the form of a statistical parametric map of the t statistic [after transformation to a SPM(Z)], thresholded at uncorrected $\mathrm{P}<0.001$. (b) The corresponding deactivations. The SPMs are displayed as maximum intensity projections viewed from the right, the back, and the top, respectively.

a

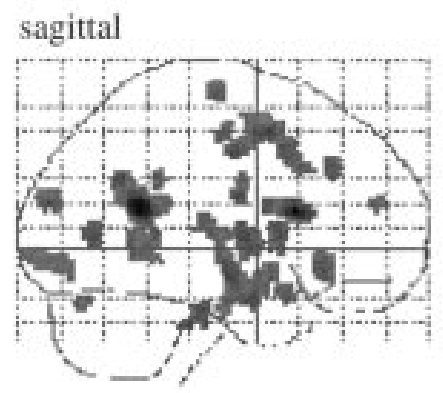

transverse

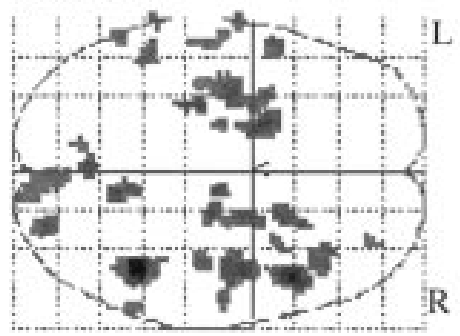

coronal

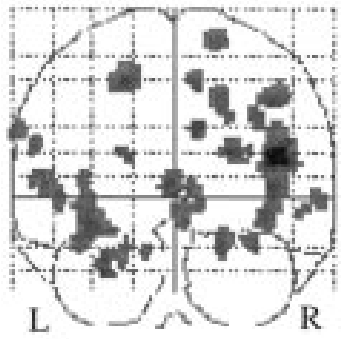

b

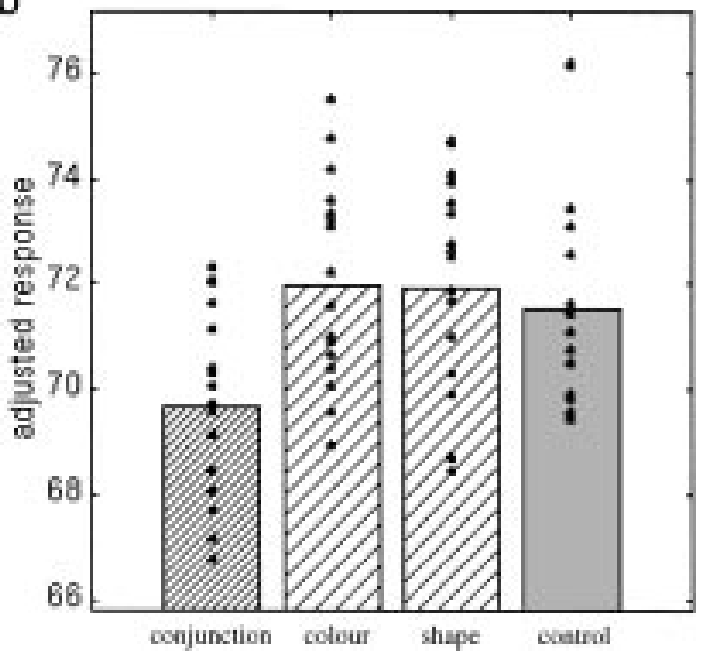

FIG. 3. (a) The relative activations associated with the single feature conditions compared to the conjunction condition. These "activations" may al so represent relative deactivations associated with the conjunction condition compared to single features. This is the case for temporal lobe structures including the left hippocampus, as shown by plotting adjusted blood flow values (b). The adjusted response, indicative of regional cerebral blood flow, is based on normalized counts and this graph shows the relative counts associated with each condition. Since the numbers represent relative counts, there are no associated units. 
ing delayed response tasks. The region of the inferior frontal gyrus activated here has also been activated in previous studies using delayed response paradigms (e.g., Goldberg et al., 1996) and there is evidence that it is involved in the encoding rather than the retrieval of visual features.

Unlike earlier studies (Roland and Gulyas, 1995; Baker et al., 1996), we did not see activation of the dorsolateral prefrontal cortex. However, both these studies required subjects to remember stimuli for much longer delays than in the present study. Our results in this respect are similar to the delayed responsestudy of J onides et al. (1993) which, using a 3-s delay, al so failed to show dorsolateral prefrontal cortex activation. Animal evidence suggests that prefrontal involvement in delayed response tasks is a function of the duration of the delay (e.g., Miller and Orbach, 1972).

A theoretical basis for our findings is provided by a series of studies by Phillips (1974) who characterized a short-term visual memory system (STVS) which is qualitatively distinct from both iconic memory and long-term visual memory. This STVS is thought to be a type of memory where recent visual events are actively visualized, is dependent on attention for active maintenance, and persists for delays of the order of $10 \mathrm{~s}$ in the absence of intervening distracters. Visual memory over longer delays than this requires converting a viewercentered representation into a structural description. The findings of the present study suggest that such a short-term posticonic visual memory system may be subsumed mainly by perceptual systems of the cerebral cortex with prefrontal activation becoming necessary only when longer term retention is required.

A noteworthy finding is relative deactivation of regions of the lateral and medial temporal cortex, including hippocampus, associated with visual memory. As discussed in the introduction, performance of DMTS paradigms is thought to critically depend on intact inferior and, more controversially, medial temporal lobe structures in animals and man. The present study is consistent with the suggestion that it may be deactivations within these regions that are essential to successful performance. Lueschow et al. (1994) described two different short-term memory mechanisms in the inferotemporal cortex, involving suppression and enhancement, respectively. The suppressive mechanism is seen in "simple stimulus repetition," a feature of tasks where animals respond automatically to any repetition of a stimulus within a trial. The DMTS paradigm, with trial unique stimuli, used here, is an example of such a task. While the physiological nature of deactivations in PET studies are incompl etely understood, it is certainly plausible that they may reflect suppressive neural mechanisms of this kind.

The direct comparisons between the three memory conditions revealed subtle feature-specific differences.
When the conjunction condition was compared with the feature only conditions, the main differences were relative deactivations in the feature conditions. The absence of specific activations associated with conjunctions was a somewhat surprising finding. In previous studies of perceptual conjunctions using sustained attentional paradigms, attention to conjunctions rather than features has been associated with relatively restricted foci of activity in the prefrontal cortex (Corbetta et al., 1991; Rees et al., 1997). While we have no clear explanation for our failure to find activation in such a region, it may be due to the relative difficulty of different conditions. In the attentional studies, the conjunction conditions are subjectively the most difficult while in the memory task of the present study, none of the subjects found the conjunction condition harder than the single features conditions. In fact the shape only condition was subjectively the most difficult and it was this condition that was associated with anterior cingulate activity.

Comparison of the feature only conditions with the conjunction condition revealed distinct patterns of activation. This is consistent with the findings of the selective/divided attention study of Corbetta et al. (1991). Activation of the left inferior frontal gyrus (BA 44) was associated with memory for color compared to conjunction and also color compared to shape. This is consistent with the argument that activation of this region in short-term memory tasks indicates that verbal rehearsal of information is taking place (Goldberg et al., 1996). The col or only condition is likely to involve more verbal rehearsal since colors are more readily verbalizable than the complex shapes in these stimuli. Memory for either perceptual feature compared to conjunction was also associated with activations in regions of the temporal lobe. However, adjusted blood flow values in these regions suggested that these effects were due to greater relative deactivation compared to the control condition for conjunction rather than greater activation for feature only conditions. A recent paper studying attentional conjunction (Rees et al., 1997), and using a parametric design which allowed the authors to report absolute deactivations, also found temporal deactivations associated with conjunctions compared to single features, suggesting this mechanism may be an important component in processing perceptual conjunctions.

Memory for shape compared to conjunction also activated a region of the right anterior cingulate, an activation also seen when shape was compared to color. Blood flow in the anterior cingulate was higher in the shape alone condition compared to each of the other conditions. This region of the right cingulate has also been implicated in tasks such as the Stroop where subjects must overcome perceptual interference in order to select a response (Pardo et al., 1990). It is 
possible that this activation represents such an effect since performance of the shape only condition requires subjects to disengage attention from the more salient attribute of the stimuli, col or (Robbins et al., 1994).

These comparisons between memory conditions also identified posterior cortical regions associated with the memory for specific perceptual dimensions. Memory for color differentially activated the left lingual gyrus which is consistent with the finding of Corbetta et al. (1991) that this region is involved in selective attention to color. Memory for shape differentially activated a region of the medial occipital gyrus and the right inferior parietal cortex and precuneus. These activations are more extensive than those reported in selective attention to shape by Corbetta et al. (1991) though it should be noted that the shapes used in the present study were much more complex. It is also possible that eye movements are contributing to the parietal activations since the discrimination of the shapes requires more local processing of detail than the discrimination of colors. The design of this study does not enable us to unconfound component processes of short-term memory, ther efore these feature-specific activations may beassociated with encoding, retention, recognition, or all of these.

The most striking feature of this study is that it indicates that a network of posterior structures can mediate short-term visual memory. These results support an account of a short-term visual memory system, analogous to the short-term visual store(Phillips, 1974; Humphreys and Bruce, 1989), dependent primarily on perceptual systems. Memory for perceptual conjunctions compared to single features did not additionally activate any regions. The relative deactivations associated with the memory for feature conjunctions observed in temporal cortex may correspond to an attentional bias effect as suggested by Rees et al. (1997). Differential activations in perceptual systems seen for memory of particular features of stimuli is consistent with the findings of Corbetta et al . (1991) that selective attention to particular perceptual features differentially activates particular posterior cortical regions. The present findings indicate that the neural substrates of object memory also reflect the perceptual properties of the stimuli.

\section{ACKNOWLEDG MENTS}

R.J .D. is supported by the Wellcome Trust. We are grateful to Karl Friston, J ohn Ashburner, Andrew Holmes, and J ean-Baptiste Poline for their work on the SPM96 technique. We also thank Richard Frackowiak, Chris Frith, and Geraint Rees for their helpful comments and discussion.

\section{REFERENCES}

Alvarez, P., Zola-Morgan, S., and Squire, L. R. 1992. Impairment of long-term memory and sparing of short-term memory in monkeys with medial temporal lobe lesions: A response to Ringo (comment). Behav. Brain Res. 52:1-5.

Alvarez, P., Zola-Morgan, S., and Squire, L. R. 1995. Damage limited to the hippocampal region produces long-lasting memory impairment in monkeys. J . Neurosci. 15:3796-3807.

Baker, S. C., Frith, C. D., Frackowiak, R. S. J ., and Dolan, R. J . 1996. Active representations of shape and spatial location in man. Cereb. Cortex 6:612-619.

Corbetta, M., Miezin, F. M., Dobmeyer, S., Shulman, G. L., and Petersen, S. E. 1991. Selective and divided attention during visual discriminations of shape, colour and speed: F unctional anatomy by positron emission tomography. J . Neurosci. 11:2388-2402.

Friston, K. J ., Frith, C. D., Liddle, P. F., and Frackowiak, R. S. J . 1991. Comparing functional (PET) images: The assessment of significant change. J . Cerebr. Blood Flow Metab. 11:690-699.

Friston, K. J ., Holmes, A. P., Worsley, K. J ., Poline, J .-B., Frith, C. D., and Frackowiak, R. S. J. 1995. Statistical parametric maps in functional imaging: A general approach. Hum. Brain Map. 2:189210.

Goldberg, T. E., Berman, K. F., Randolph, C., Gold, J. M., and Weinberger, D. R. 1996. I solating the mnemonic component in spatial delayed response: A controlled PET ${ }^{15} \mathrm{O}-$ labeled water regional cerebral blood flow study in humans. Neuroimage 3:69-78.

Gross, C. G. 1973. Visual functions of the inferotemporal cortex. In Handbook of Sensory Physiology (R. J ung, Ed.), pp. 451-482. Springer, Berlin.

Horel, J. A., Pytko-J oiner, D. E., Voytko, M. L., and Salisbury, K. 1987. The performance of visual tasks while segments of the inferotemporal cortex are suppressed by cold. Behav. Brain Res. 23:29-42.

Humphreys, G. W., and Bruce, V. 1989. Visual Cognition: Computational, Expermantal and Neuropsychological Perspectives. Lawrence Erlbaum, Hove, UK.

J onides, J ., Smith, E. E., Koeppe, R. A., Awh, E., Minoshima, S., and Mintun, M. A. 1993. Spatial working memory in humans as revealed by PET. Nature 363:623-625.

Lueschow, A., Miller, E. K., and Desimone, R. 1994. Inferior temporal mechanisms for invariant object recognition. Cerebr. Cortex 5:523531.

Meunier, M., Bachevalier, J ., Mishkin, M., and Murray, E. A. 1993. Effects on visual recognition of combined and separate ablations of the entorhinal and perirhinal cortex in rhesus monkeys. J . Neurosci. 13:5418-5432.

Miller, M. H., and Orbach, J . 1972. Retention of spatial alternation following frontal lobe resections in stump-tailed macaques. Neuropsychologia 10:291-298.

Milner, B. 1970. Memory and medial temporal regions of the brain. In Biology of Memory (K. H. Pribram and D. E. Broadbent, Eds.) Academic Press, New York.

Mishkin, M. 1982. A memory system in the monkey. Phil. Trans. R. Soc. London B298:85-92.

Mishkin, M., and Murray, E. A. 1994. Stimulus recognition. Curr. Opin. Neurobiol . 4:200-206.

Murray, E. A. 1992. Medial temporal lobe structure contribute to recognition memory: The amygdaloid complex versus the rhinal cortex. In The Amygdala: Neurobiological Aspects of Emotion, Memory and Mental Dysfunction (J . P. Aggleton, Ed.), pp. 453-470, Wiley, New York.

Owen, A. M., Sahakian, B. J ., Semple, J ., Polkey, C. E., and Robbins, T. W. 1995. Visuo-spatial short term recognition memory and learning after temporal lobe excisions, frontal lobe excisions or amygdalo-hippocampectomy in man. Neuropsychol ogia 33:1-24. 
Owen, A. M., Milner, B., Petrides, M., and Evans, A. C. 1996. Memory for object features versus memory for object locations: A positron emission tomography study of encoding and retrieval processes. Proc. Natl. Acad. Sci. USA 93:9212-9217.

Pardo, J . V., Pardo, P. J ., J aner, K. W., and Raichle, M. E. 1990. The anterior cingulate cortex mediates processing selection in the Stroop attentional conflict paradigm. Proc. Natl. Acad. Sci. 87:256259.

Phillips, W. A. 1974. On the distinction between sensory storage and short term visual memory. Percept. Psychophys. 16:283-290.

Rees, G., Frackowiak, R. S. J ., and Frith, C. D. 1997. Two modulatory effects of attention mediating object categorisation in human cortex. Science(in press).

Robbins, T. W., J ames, M., Owen, A. M., Sahakian, B. J ., Mcl nnes, L., and Rabbitt, P. 1994. Cambridge Neuropsychological Battery
(CANTAB): A factor analytic study of a large sample of normal elderly volunteers. Dementia 5:266-81.

Roland, P. E., and Gulyas, B. 1995. Visual memory, visual imagery and visual recognition of large field patterns by human brain: Functional anatomy by positron emission tomography. Cerebr. Cortex 1:79-93.

Talairach, J ., and Tournoux, P. 1988. Coplanar Stereotactic Atlas of theH uman Brain. George Thieme Verlag, Stuttgart.

Zola-Morgan, S., and Squire, L. R. 1985. Medial temporal lesions in monkeys impair memory on a variety of tasks sensitive to human amnesia. Behav. Neurosci. 99:22-34.

Zola-Morgan, S., Squire, L. R., Amaral, D. G., and Suzuki, W. 1989. Lesions of perrhinal and parahippocampal cortex that spare the amygdala and hippocampal formation produce severe memory impairment. J . Neurosci. 9:4355-4370. 